\title{
Molecular mechanism of phosphate steering for DNA binding, cleavage localization, and substrate release in nucleases
}

\author{
Elisa Donati, ${ }^{1}$ Pietro Vidossich, ${ }^{1}$ Marco De Vivo ${ }^{1 *}$
}

1. Laboratory of Molecular Modeling and Drug Discovery, Istituto Italiano di Tecnologia, via Morego 30, 16163 Genova, Italy

Corresponding authors:

Marco De Vivo E-mail: marco.devivo@iit.it

\section{Supporting Information}




\section{Table of Contents}

\section{Supplementary Figures}

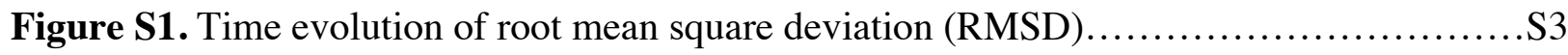

Figure S2. Graphs reporting the correlation between $\operatorname{Arg} 100$ and +1 phosphate positions......S4

Figure S3. Graphs reporting the distances between Arg104, Lys132 and the 5'-flap............S5

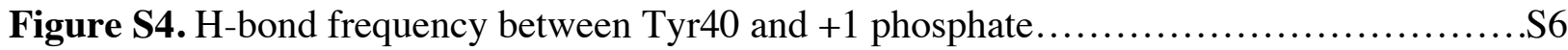

Figure S5. Comparison between $\alpha^{\text {sld1 }}, \alpha^{\mathrm{dld} 2}$ dihedral angles from $w t$ and RRRK-A/-E.........S7

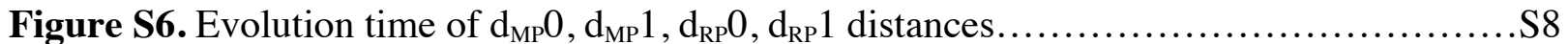

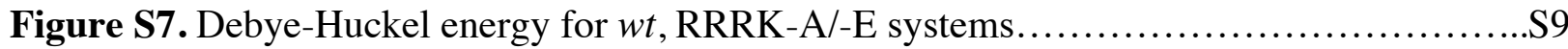

Figure S8. Graphs reporting differences between $5 \mathrm{PO}$ and $5 \mathrm{OH}$ systems................... 10

Figure S9. Energy values for the dihedral angle $(\delta)$ oh Tyr40 for $w t$, RRRK-A/-E systems....S11

Figure S10. pMtD free-energy surface (FES) of 5PO, 5OH systems.......................

Figure S11. Confined well-tempered metadynamics FES $\ldots \ldots \ldots \ldots \ldots \ldots \ldots \ldots \ldots \ldots \ldots . . . . \ldots \ldots$

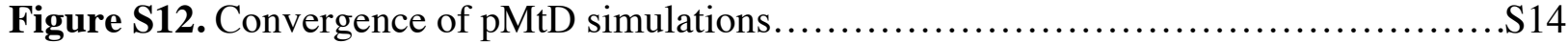

Figure S13. Convergence of the leaving group departure.............................. 15

Figure S14. Comparison of free-energy profiles for hFEN1-5' flap system.................S16

Figure S15. Comparison of free-energy profiles for both hFEN1-5'PO/5' OH systems..........S17

Scheme S1. Scheme of the catalytic steps for hFEN1 catalysis on $5 \mathrm{PO}$ substrate............S18 
A
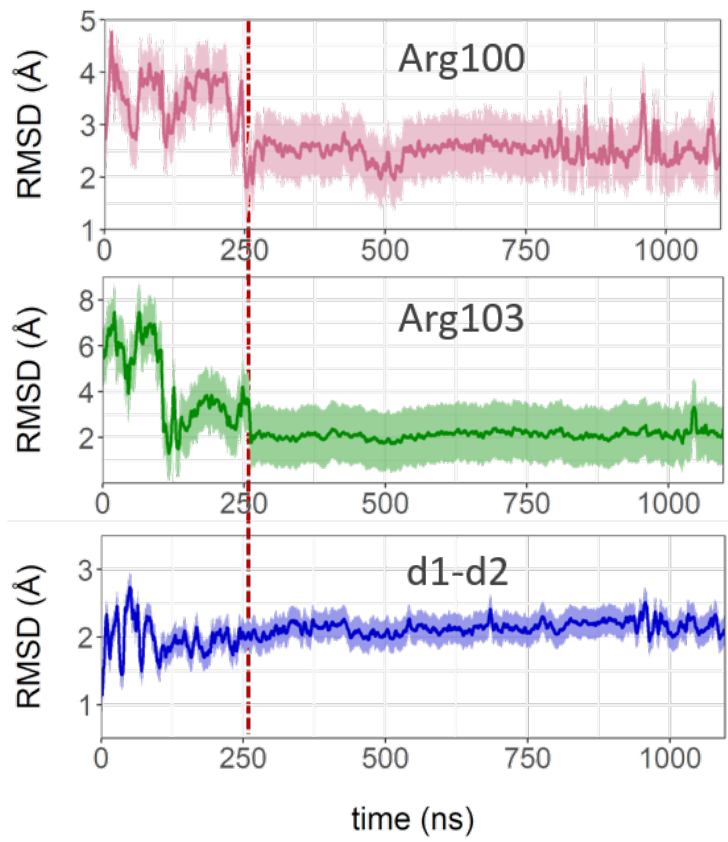

B

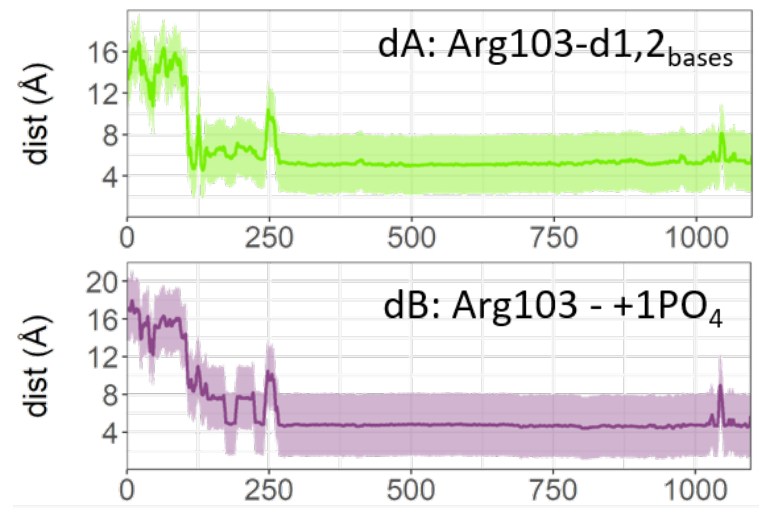

C

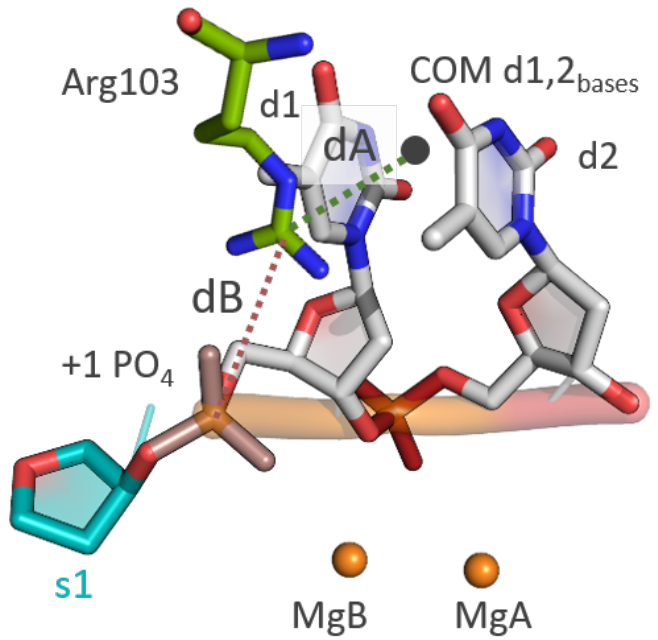

Figure S1. MD simulation of the $w t$ system. (A) Time evolution of the root mean square deviation (RMSD) for the heavy atoms of $\operatorname{Arg} 100, \operatorname{Arg} 103$ and nucleotides d 1 and d2, using as reference the reactant state crystal structure (PDB ID 5UM9). The red dashed line marks the reorganization of Arg100 and Arg103. (B) Time evolution of distances dA and $\mathrm{dB} . \mathrm{dA}$ is the distance between the center of mass (C.O.M.) of the heavy atoms of the guanidinium group of $\operatorname{Arg} 103$ and the C.O.M. of the heavy atoms of both $\mathrm{d} 1$ and $\mathrm{d} 2$ nucleobases. $\mathrm{dB}$ is the distance between the C.O.M. of the heavy atoms of guanidinium group of Arg103 and the C.O.M. of the +1 phosphate. After $\sim 250 \mathrm{~ns} \mathrm{dA}$ and $\mathrm{dB}$ stabilize around $4.5 \AA$ and $5 \AA$, respectively. (C) Schematic representation of nucleotides $\mathrm{d} 2$, d1 and main chain atoms of s1 together with the +1 phosphate (highlighted in pink), the Arg103 residue (in licorice, colored in green), the catalytic Mg ions (as spheres) and the distances $\mathrm{dA}$ and $\mathrm{dB}$ (dashed lines). The C.O.M. of the nucleobases of $\mathrm{d} 1$ and $\mathrm{d} 2$ is represented as a dark grey sphere. 
A

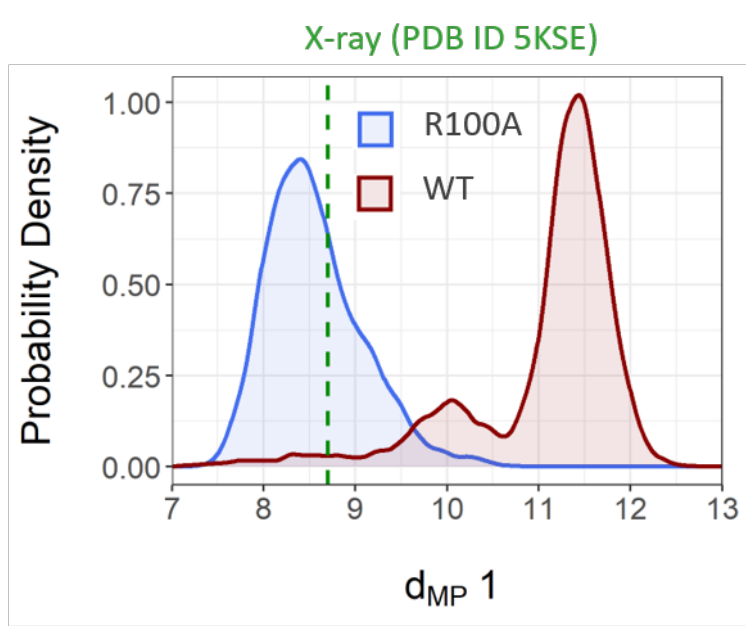

B
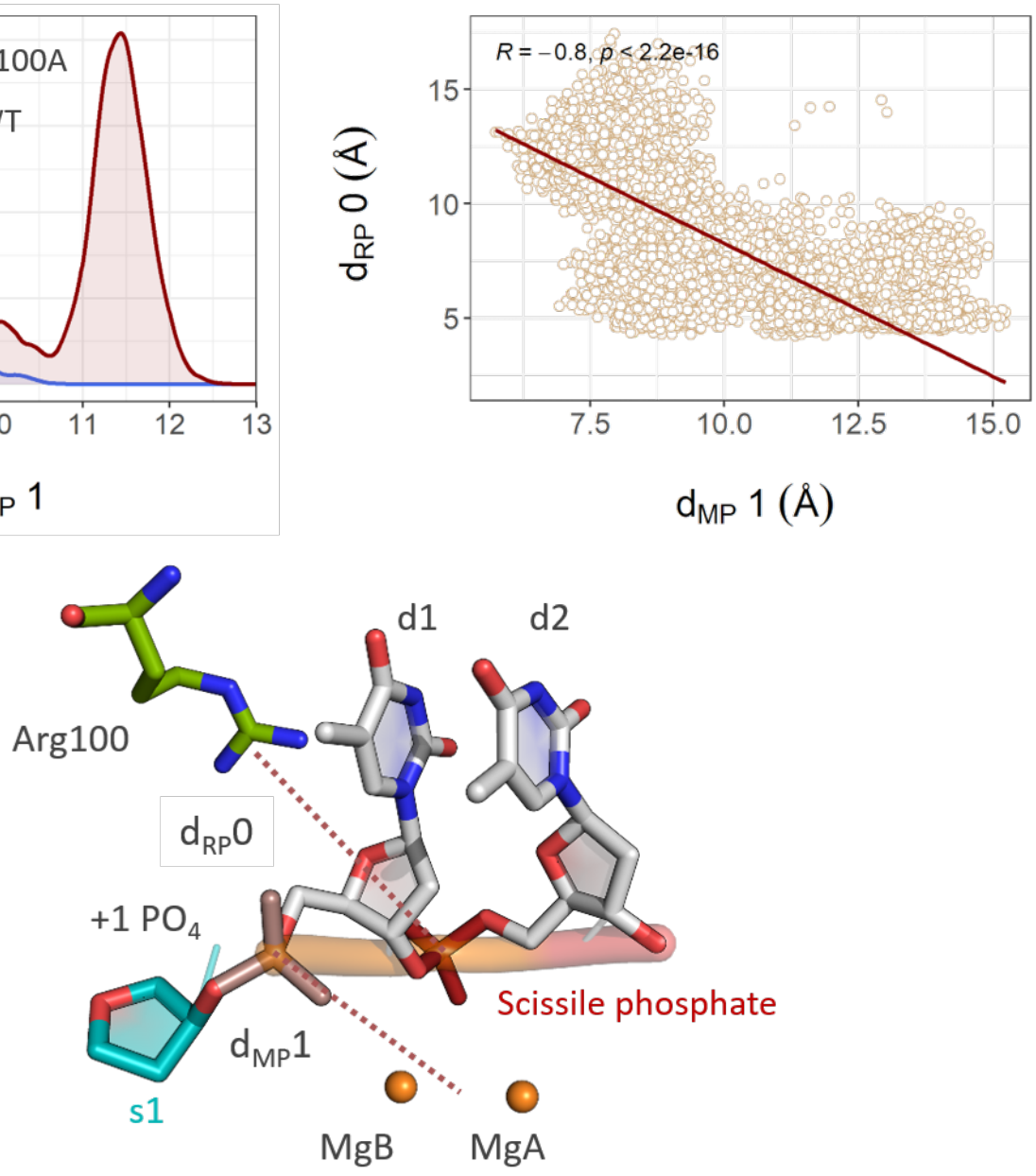

Figure S2. (A) Probability density of the distance $\mathrm{d}_{\mathrm{MP}} 1$ for the $w t$ and R100A systems during MD simulations. $\mathrm{d}_{\mathrm{MP}} 1$ is the distance between the C.O.M. of the +1 phosphate and the C.O.M. of the catalytic metal ions $\mathrm{MgA}$ and $\mathrm{MgB}$. The green dashed line marks the crystallographic value of $\mathrm{d}_{\mathrm{MP}} 1$ (PDB ID 5KSE) where Arg 100 is mutated to Ala. (B) Scatter plot of distances $\mathrm{d}_{\mathrm{MP}} 1$ and $\mathrm{d}_{\mathrm{RP}} 0$ (where $\mathrm{d}_{\mathrm{RP}} 0$ represents the distance between the C.O.M. of the heavy atoms of guanidinium group of Arg100 and the C.O.M. of the scissile phosphate) collected from the MD simulations of the $w t$ and RKKK-A/-E systems. The correlation has been calculated using Pearson's method. (C) Schematic representation of nucleotides $\mathrm{d} 2, \mathrm{~d} 1$ and main chain atoms of $\mathrm{s} 1$, together with the +1 and the scissile phosphates (highlighted in pink and red, respectively), $\operatorname{Arg} 100$ (in green licorice), the catalytic Mg ions (as orange sphere). The distances $\mathrm{d}_{\mathrm{MP}} 1$ and $\mathrm{d}_{\mathrm{RP}} \mathrm{O}$ are shown as dashed lines. 
A
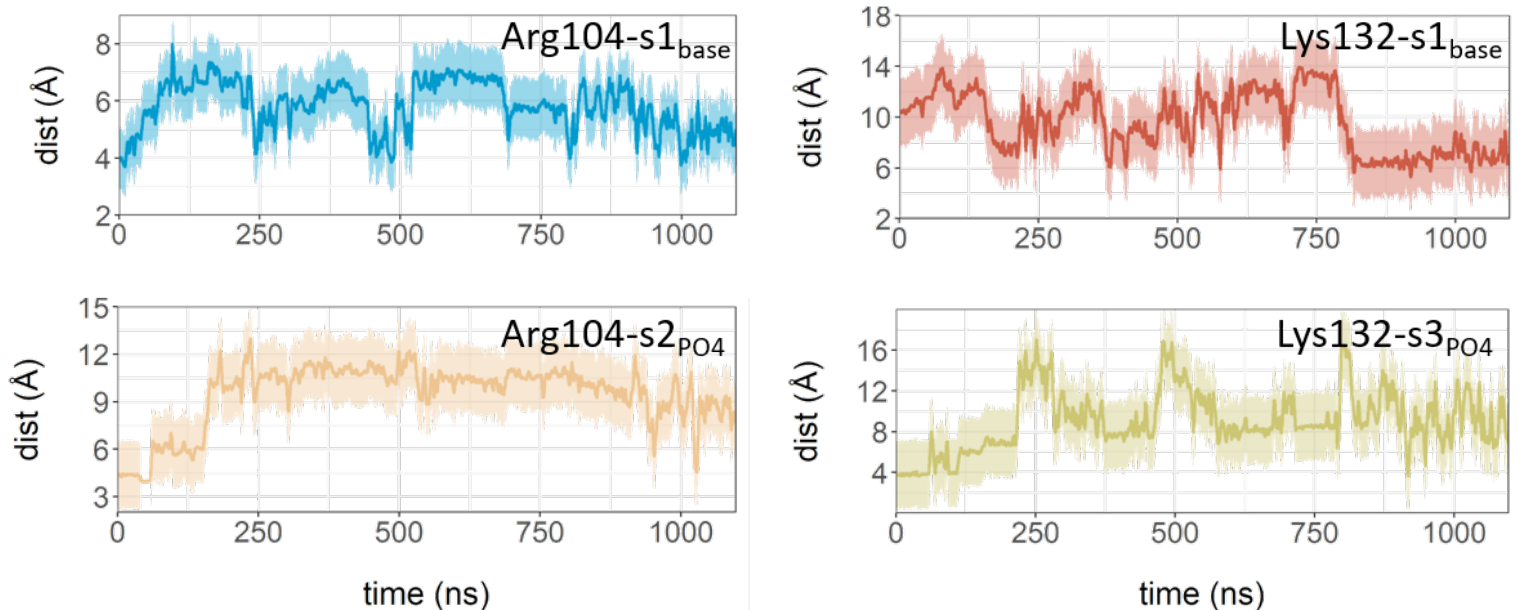

B

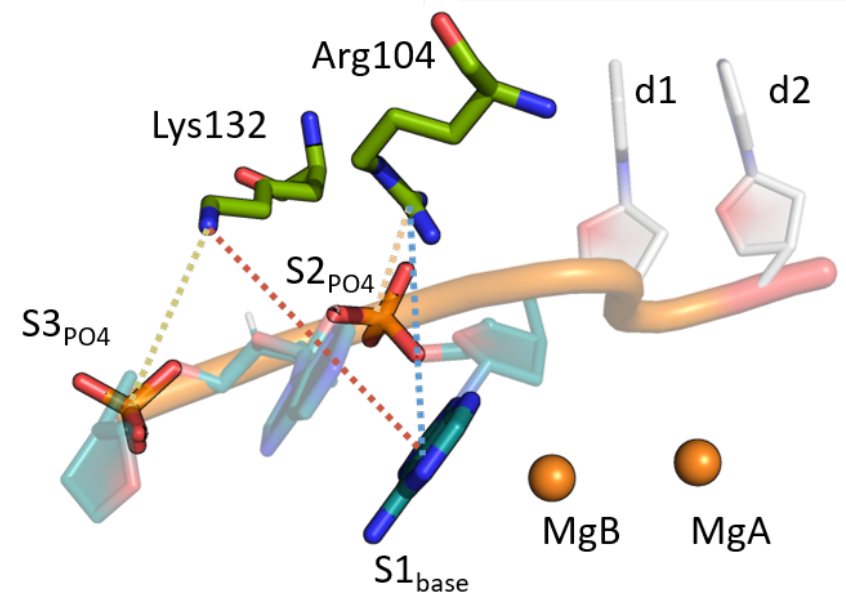

Figure S3. (A) On the left, the distance between the C.O.M. of the guanidinium group of Arg104 and the C.O.M. of the nucleobase of nucleotide s1 (blue line), and the distance between the C.O.M. of the guanidinium group of Arg104 and the C.O.M. of the phosphate between nucleotides s1 and s2 (orange line). On the right, the distance between the C.O.M. of the amino group of Lys132 and the C.O.M. of the nucleobase of nucleotide s1 (red line), and the distance between the C.O.M. of the amino group of Lys132 and the C.O.M. of the phosphate between nucleotides s2 and s3 (yellow line). (B) Representative snapshot from the MD simulation of the $w t$ system showing Arg104, Lys132 (green licorice), nucleotides d2 to s3 along the 5' strand of DNA and the two catalytic metal ions (as orange spheres). 

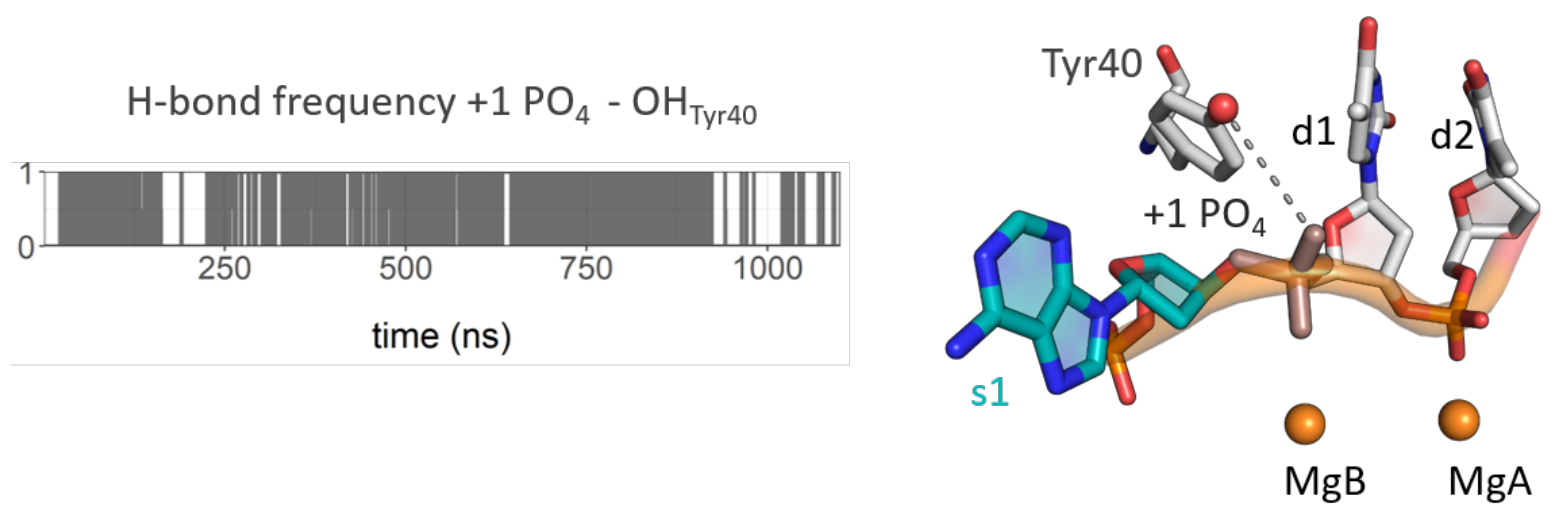

Figure S4. MD simulations of the $w t$ system. On the left, occupancy of the H-bond between the OH group of Ty40 side chain and the +1 phosphate. The requirements for the formation of the H-bond were: distance donor-acceptor < $3.5 \AA$ and the cutoff angle $<25^{\circ}$. On the right, representative snapshot showing Tyr40 (licorice, with the OH group as red sphere), nucleotides d2, d1 and s1 along the 5'-strand of DNA and the two catalytic metal ion (as orange spheres). 


\section{A}

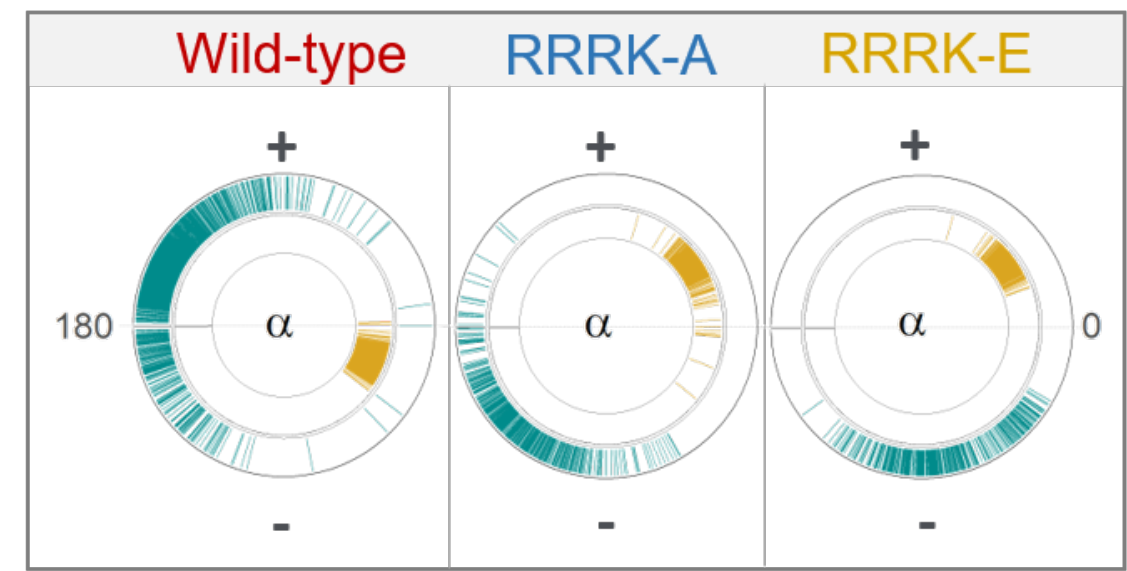

B Snapshot from MD simulation (RRRK-E system)

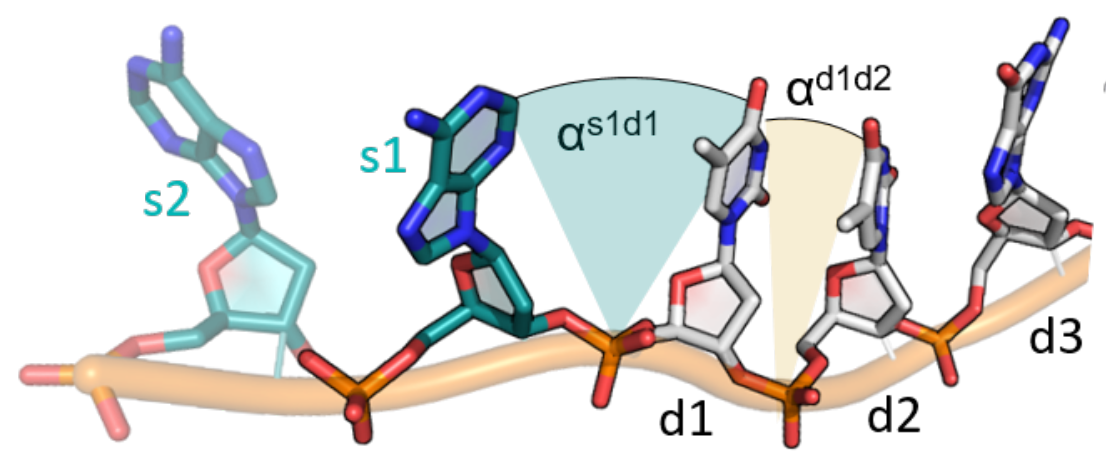

Figure S5. A) Representation of the $\alpha^{\mathrm{sld} 1}, \alpha^{\mathrm{d} 1 \mathrm{~d} 2}$ dihedral angles calculated during MD simulations of the $w t$, RRRKA and RRRK-E systems, defined as the angle between the planes of the nucleobases of nucleotides d1 and s1 ( $\left.\alpha^{\text {sld1 }}\right)$, and $\mathrm{d} 1$ and $\mathrm{d} 2\left(\alpha^{\mathrm{d} 1 \mathrm{~d} 2}\right)$ within the 5' strand. B) Representative snapshot from the MD simulation of the RRRK-E system showing the non-inverted orientation of nucleotides $\mathrm{d} 3$ to s2. The dihedral angles $\alpha^{\text {sld1 }}$ and $\alpha^{\mathrm{d} 1 \mathrm{~d} 2}$ are also represented. 
A

Wild-type

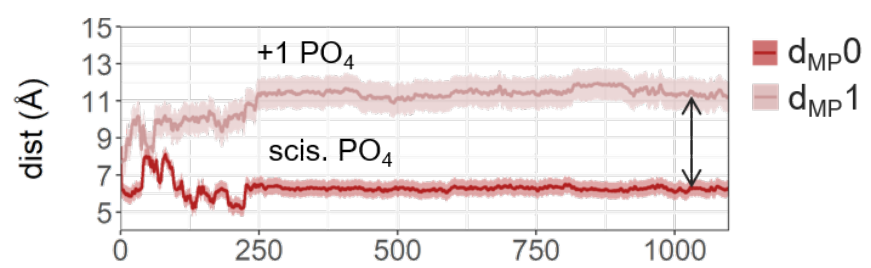

RRRK-A
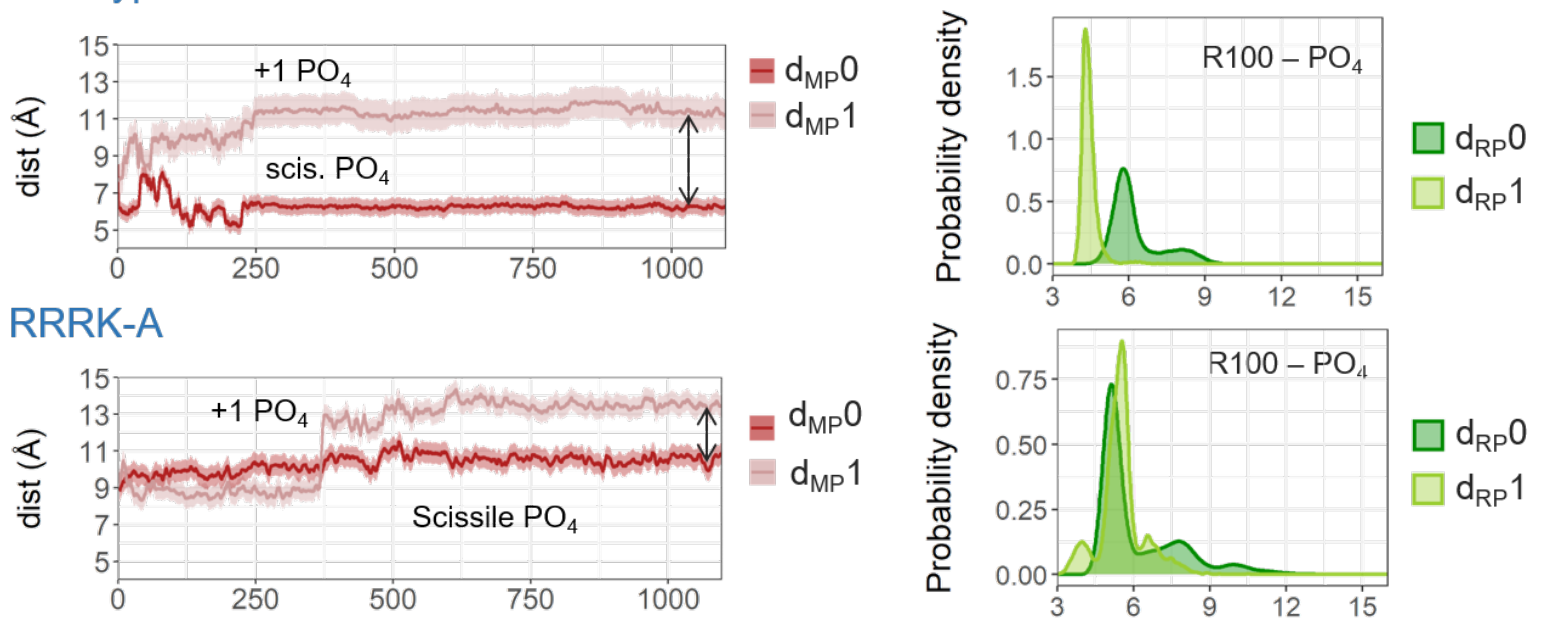

RRRK-E
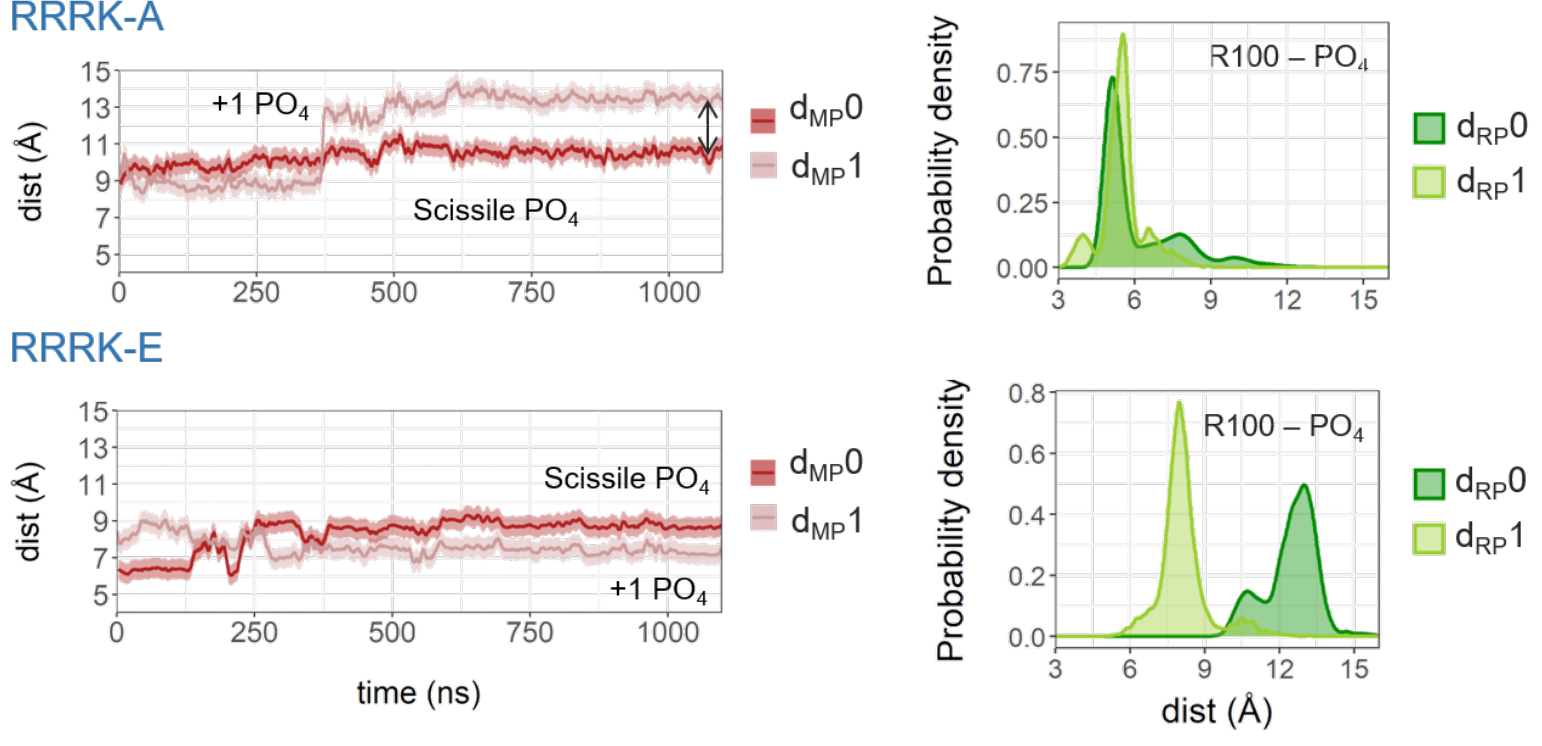

B

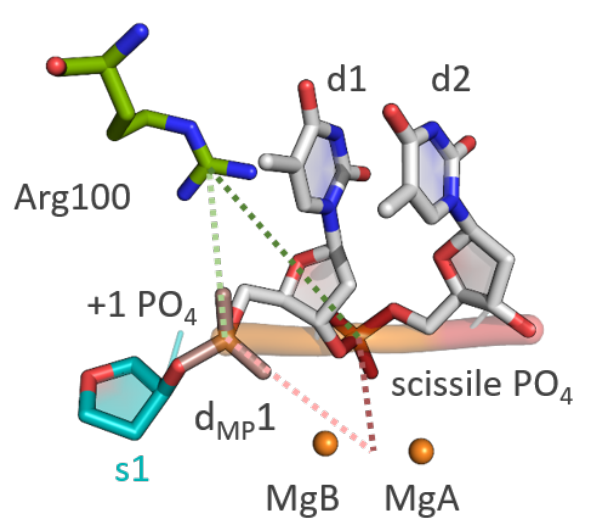

Figure S6. (A) On the left, time evolution of distances $\mathrm{d}_{\mathrm{MP}} 0$ (red line) and $\mathrm{d}_{\mathrm{MP}} 1$ (pink line); on the right, probability density of $\mathrm{d}_{\mathrm{RP}} 0$ (green line) and $\mathrm{d}_{\mathrm{RP}} 1$ (chartreuse line) from MD simulations of the $w t$, RRRK-A and RRRK-E systems. $\mathrm{d}_{\mathrm{MP}} 0$ and $\mathrm{d}_{\mathrm{MP}} 1$ are the distances between the the C.O.M. of the catalytic MgA and MgB ions and the C.O.M. of the scissile and +1 phosphates, respectively. $\mathrm{d}_{\mathrm{RP}} 0$ and $\mathrm{d}_{\mathrm{RP}} 1$ are the distances between the C.O.M. of the guanidinium group of $\operatorname{Arg} 100$ and the C.O.M. of the scissile and +1 phosphates, respectively. (B) Representation of nucleotides d2, d1 and s1 together with the +1 and scissile phosphates (highlighted in pink and red, respectively), $\operatorname{Arg} 100$ (green licorice), the catalytic $\mathrm{Mg}$ ions (as orange sphere) and the distances $\mathrm{d}_{\mathrm{RP}} 0, \mathrm{~d}_{\mathrm{RP}} 1, \mathrm{~d}_{\mathrm{MP}} 0$ and $\mathrm{d}_{\mathrm{MP}} 1$. 

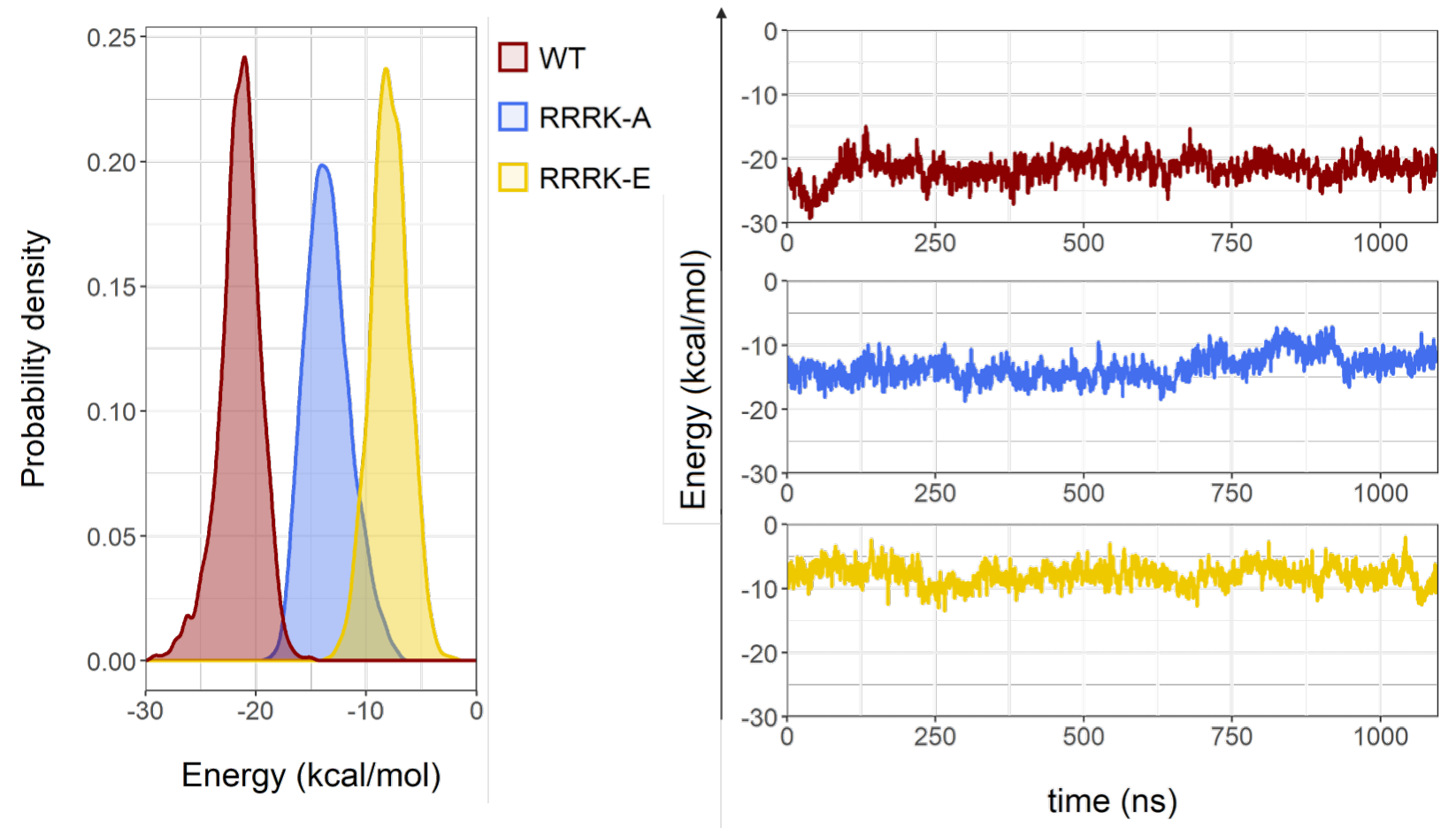

Figure S7. (Left) Probability density of the Debye-Huckel interaction energy between the 5'flap DNA substrate and the $w t$ and RRRK-A/-E hFEN1 enzymes. The Debye-Huckel interaction energy was calculated using Plumed2.6, setting the temperature to $310 \mathrm{~K}$, the ionic strength to $1 \mathrm{M}$ and the dielectric constant of the solvent to 80 . (Right) Time evolution of the Debye-Huckel interaction energy during the simulations of the $w t$ (dark red line), RRRK-A (blue) and RRRK-E (yellow) systems. 
A
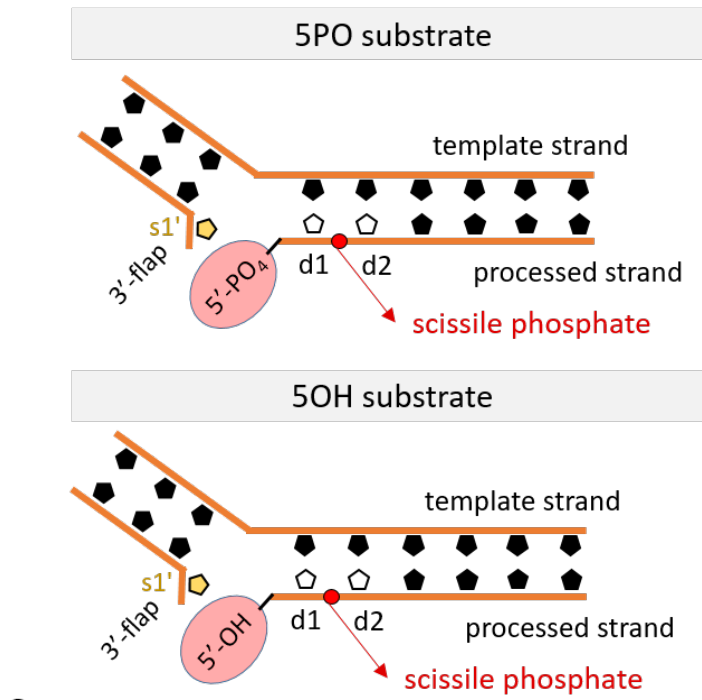

C

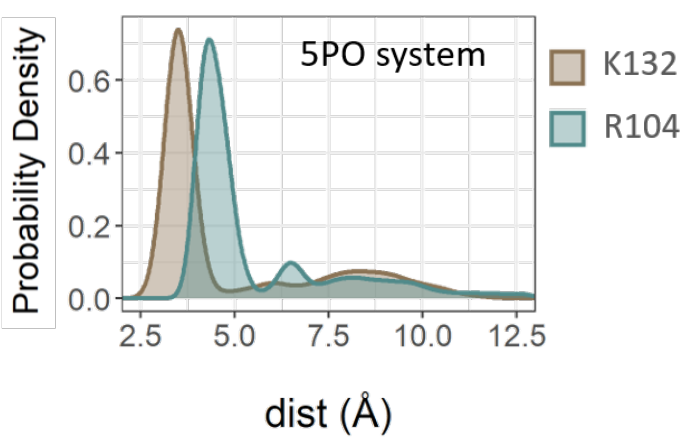

B

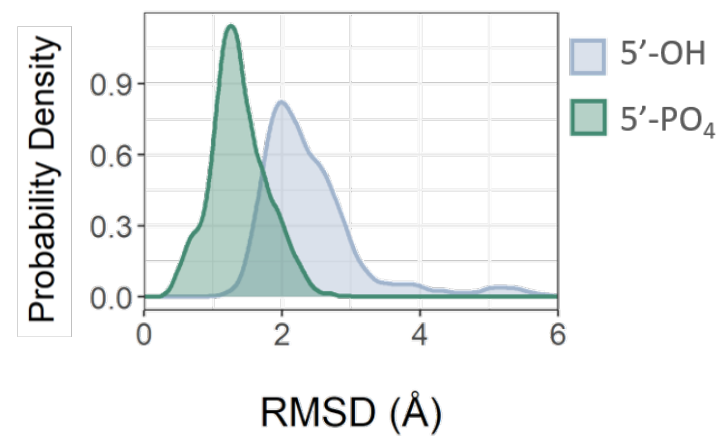

D

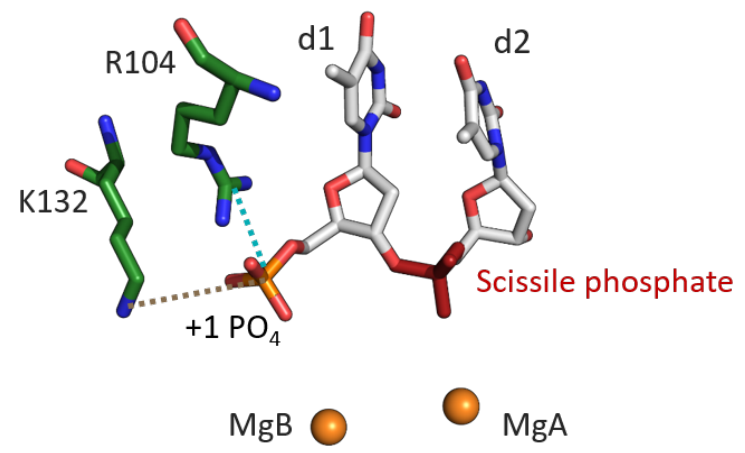

Figure S8. (A) Schematic representation of the exonucleolytic DNA substrates (i.e. without the 5'-flap), ending with either a 5'-monophosphate (top) or 5'-OH group (bottom). They correspond to the DNA substrates of the 5PO and $5 \mathrm{OH}$ systems. The scissile phosphate as for the optimal double-flap DNA substrate is indicated with a red arrow. (B) Probability density of the RMSD values for the heavy atoms of the terminal 5'-nucleotide during the simulations of the 5PO and 5OH systems. (C) Probability density of the distance between the C.O.M. of the +1 phosphate (i.e. the terminal 5'-phosphate) and the C.O.M. of the heavy atoms of the guanidinium group of Arg 104 (cyan line) and the distance between the C.O.M. of the +1 phosphate and the C.O.M. of the heavy atoms of the amino group of Lys 132 (brown). (D) Representative snapshot from the MD simulation of the 5PO system showing Arg 104, Lys132 (in green licorice), nucleotides $\mathrm{d} 1$ and $\mathrm{d} 2$ along the 5'-strand of DNA and the two catalytic metal ions (as orange spheres). 
A

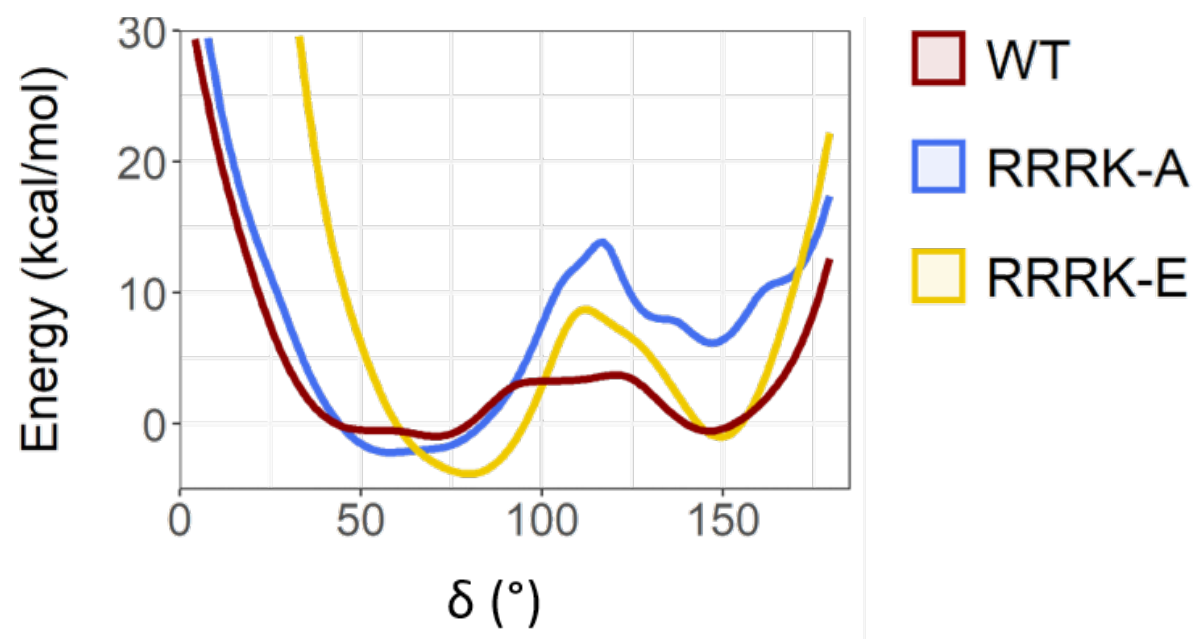

Threaded state

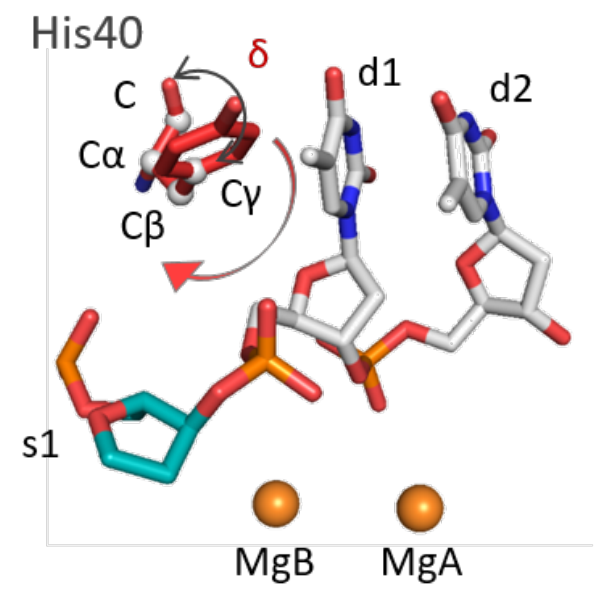

Reactant state

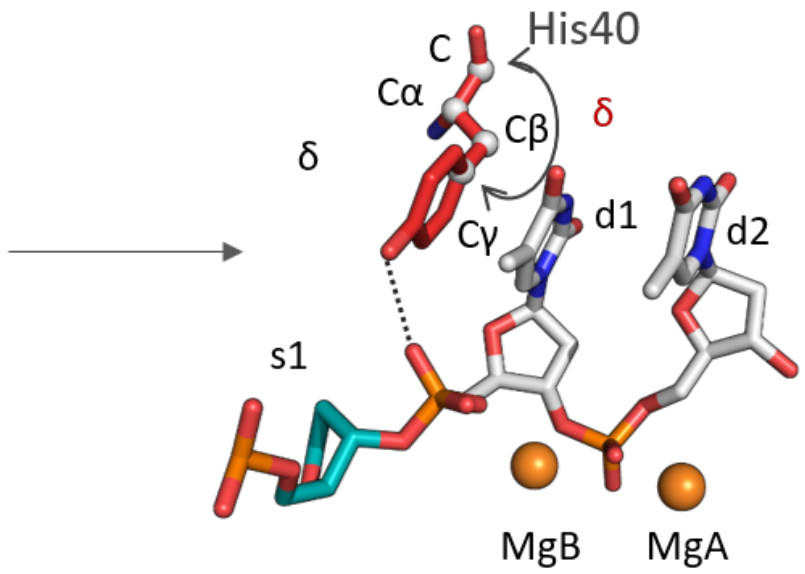

Figure S9. (A) Free energy profile (in $\mathrm{kJ} \mathrm{mol}^{-1}$ ) corresponding to the dihedral angle ( $\delta$ ) for the $w t, \mathrm{RRRK}-\mathrm{A} /-\mathrm{E}$ systems, as reconstructed from the pMtD simulations reweighting the histograms of the sampled distributions and converting them into free energy values, as implemented in Plumed. (B) Representation of the two conformations assumed by Tyr40 side chain and corresponding to the two minima in the free energy profiles shown in (A). On the left, Tyr40 is not able to form H-bond interactions with the phosphate, while on the right (i.e. in the reactant state) Tyr40 side chain has rotated and thus can interact with the phosphate (dashed black line). The atoms taken into account to calculate the dihedral angle $\delta$ (i.e. $C, C \alpha, C \beta$ and $C \gamma$ ) are shown as white spheres. 


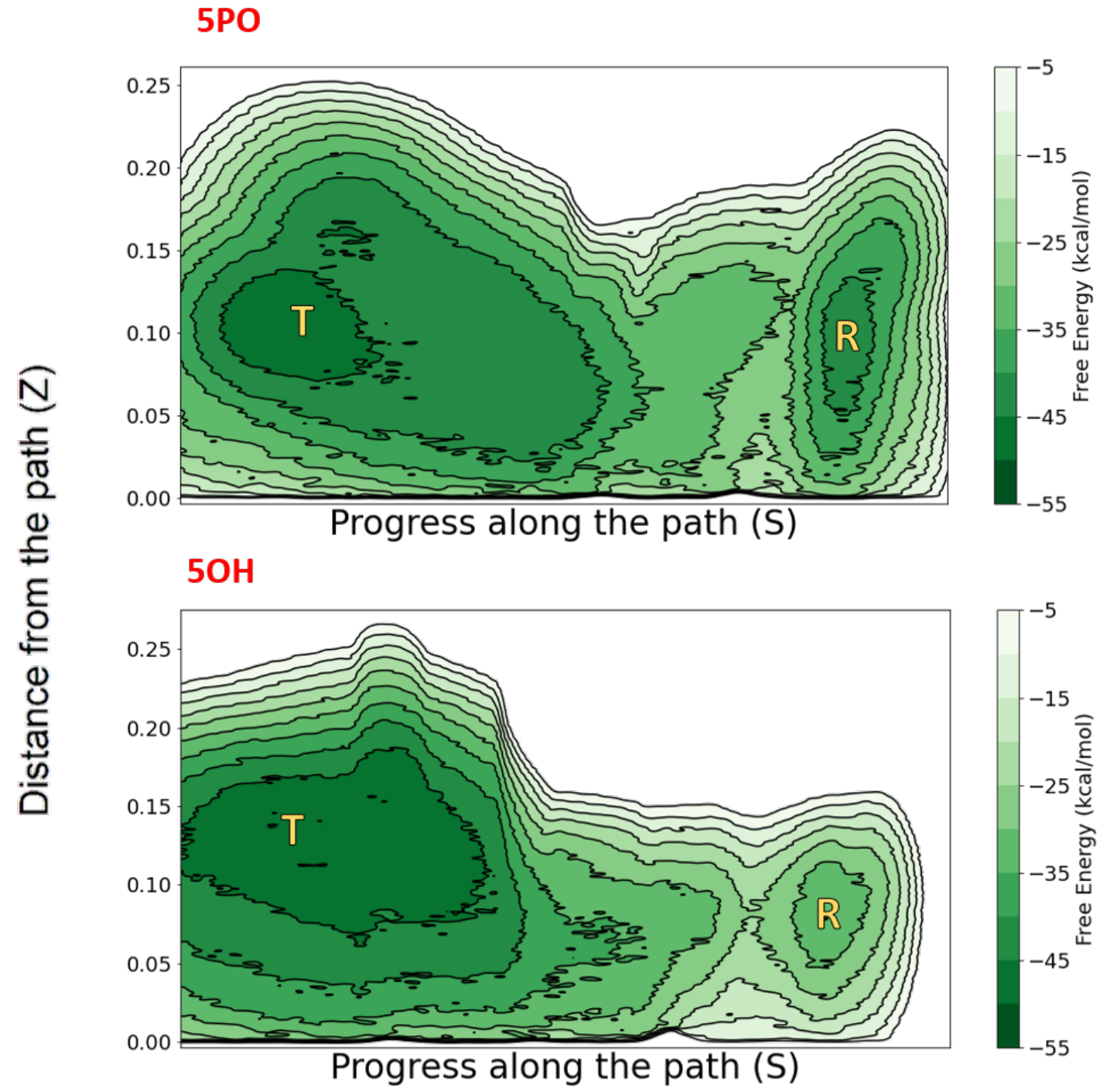

Figure S10. pMtD free-energy landscape of the 5PO and 5OH systems, where the threaded (T) and reactant (R) states are indicated. 

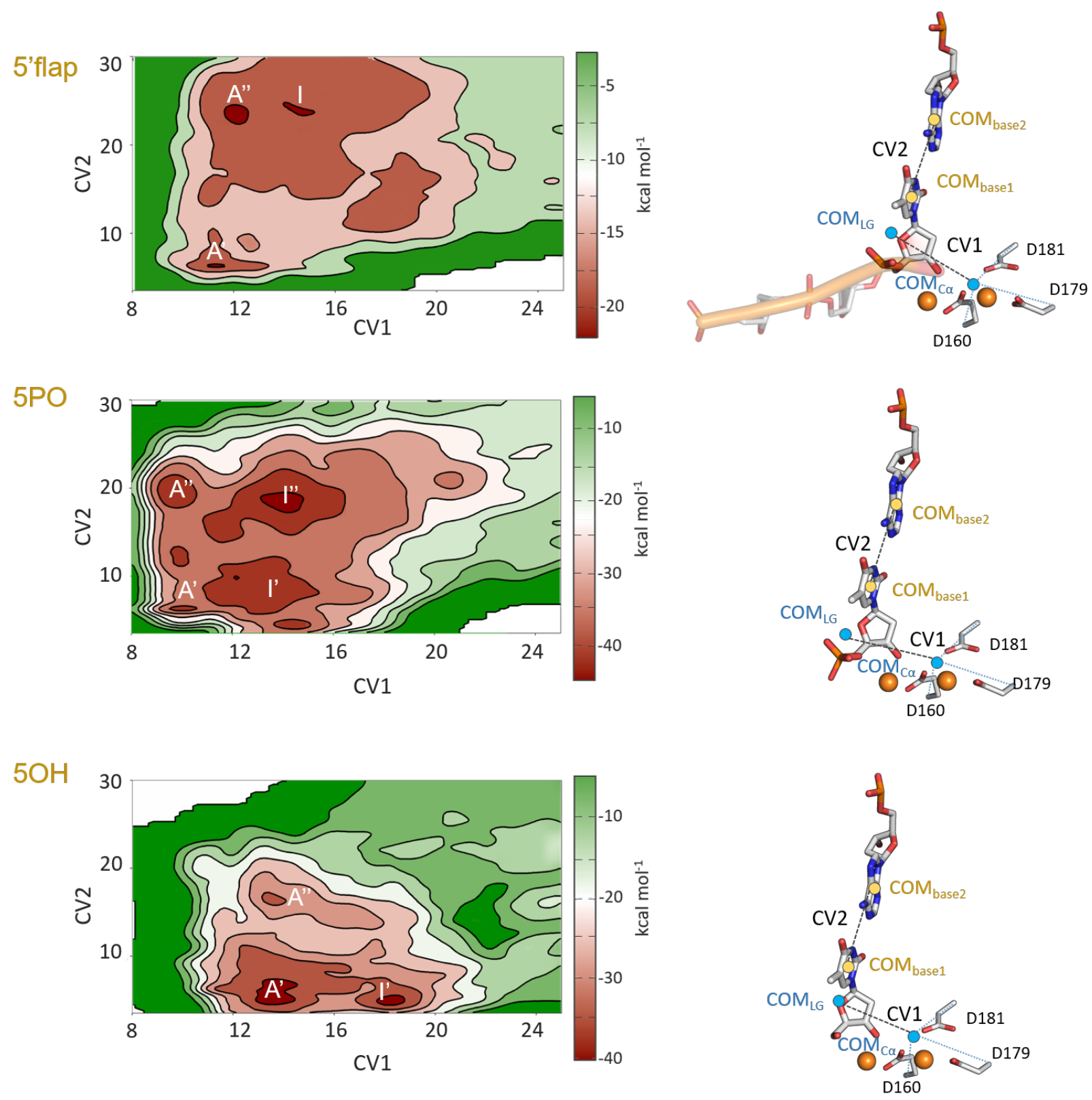

Figure S11. Free energy landscape for the leaving group release in the 5'-flap, 5PO and 5OH systems from confined well-tempered metadynamics simulations. The minima are indicated with white letters. A representation of the collective variables CV1 and CV2 is also shown for the three systems. CV1 is the distance between the C.O.M. of the heavy atoms of the nucleotide leaving group and the C.O.M. of the C $\alpha$ of Glu160, Asp179, Asp181 in the first coordination shell of ions $\mathrm{MgA}$ and $\mathrm{MgB}$ (blue circles). CV2 is the distance between the C.O.M. of the nucleobase of $\mathrm{d} 1$ and the complementary nucleotide (yellow circles). 


\section{Convergence of the pMtD}
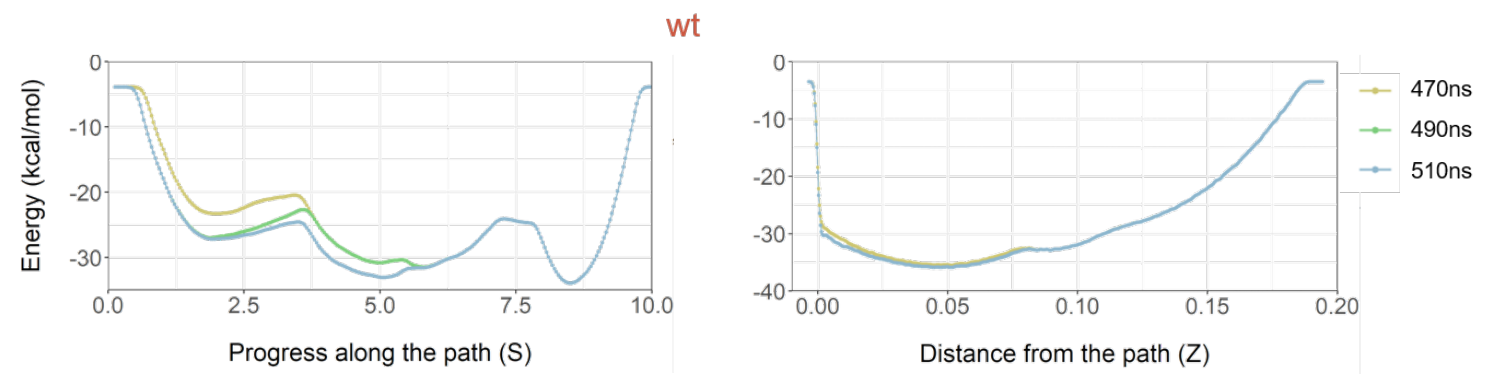

RRRK-A
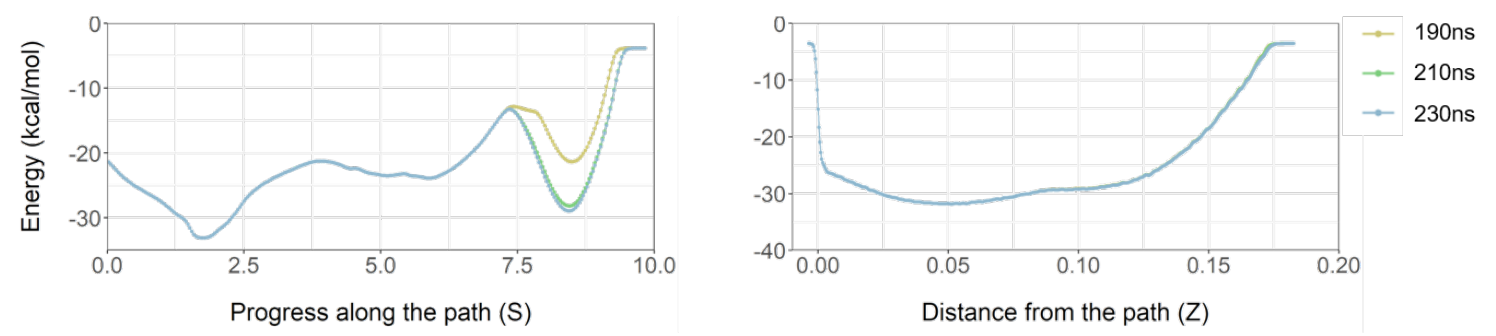

RRRK-E
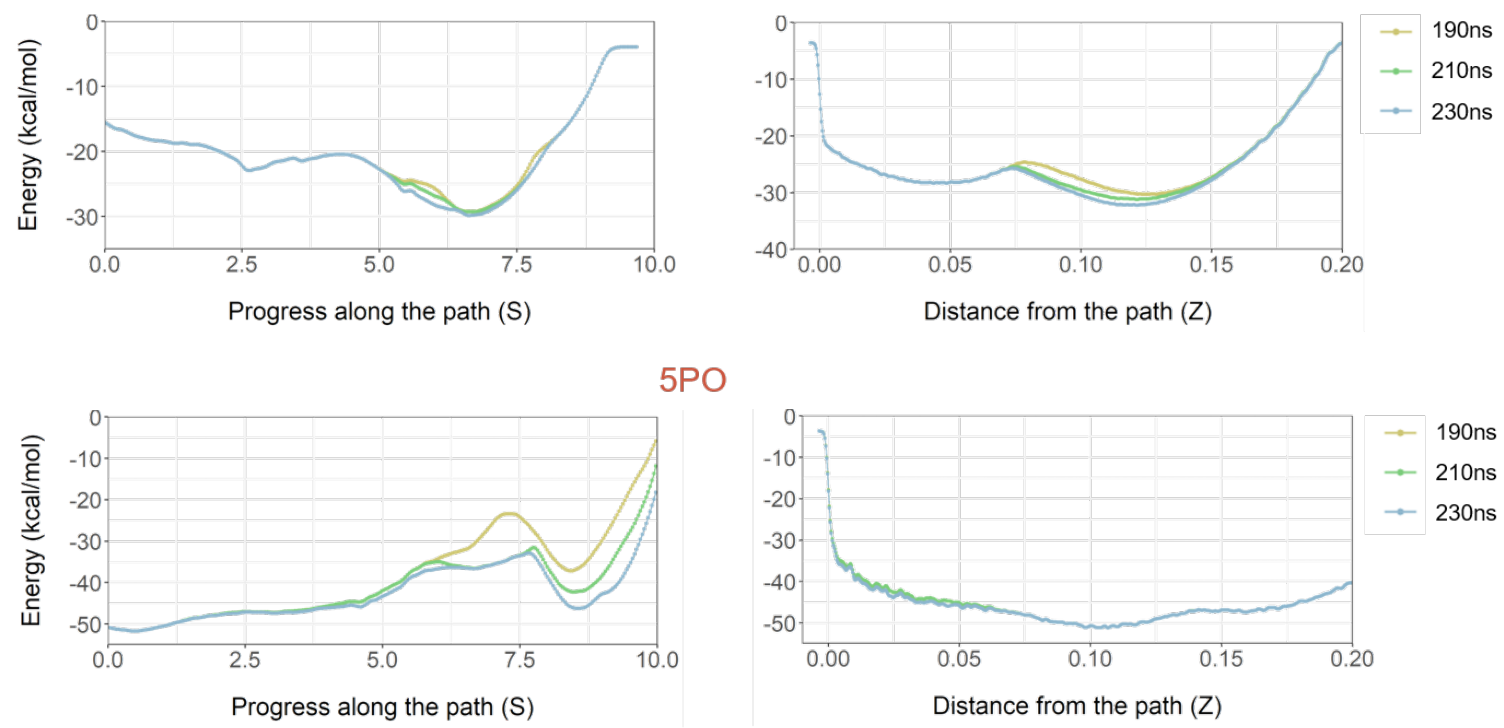

$5 \mathrm{OH}$
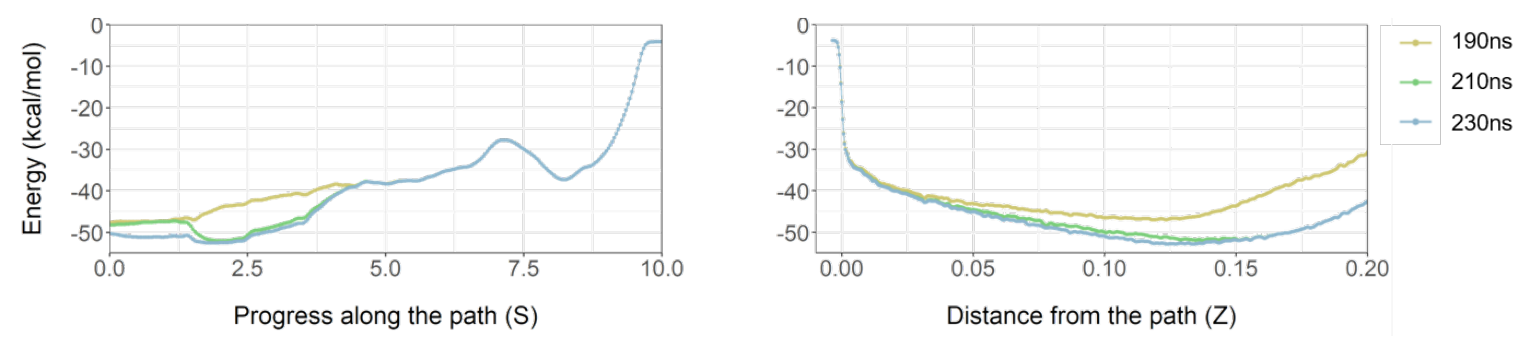

Figure S12. Convergence of the free energy profiles from the pMtD simulations of the $w t$, RRRK-A/-E, 5PO and $5 \mathrm{OH}$ systems. The projections of the free energy on the path variables $\mathrm{S}$ (left panels) and $\mathrm{Z}$ (right panels) are shown during the progress of the metadynamics simulations. 


\section{Convergence of the leaving group departure}

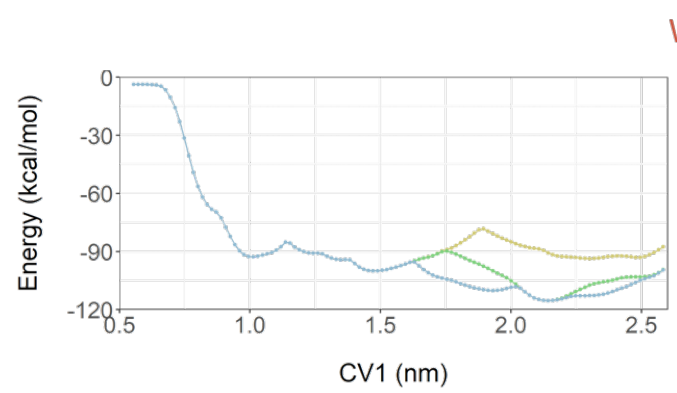

Wt-flap
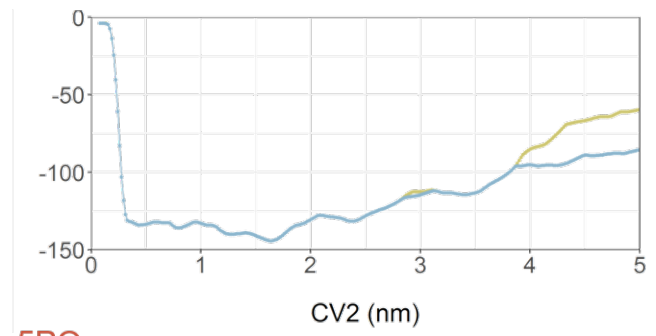

$\rightarrow 1.9^{*} 10^{8}$ steps

$\rightarrow 2.1^{\star} 10^{8}$ steps

$\rightarrow 2.3^{*} 10^{8}$ steps

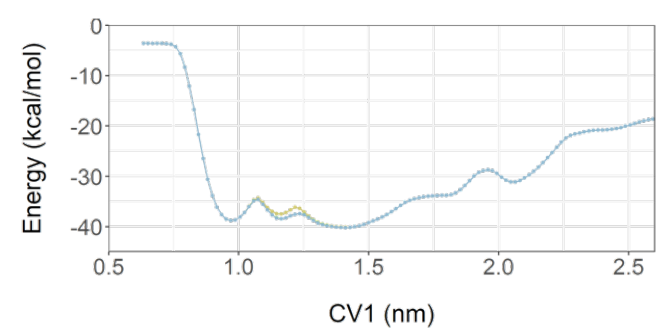

$5 \mathrm{PO}$
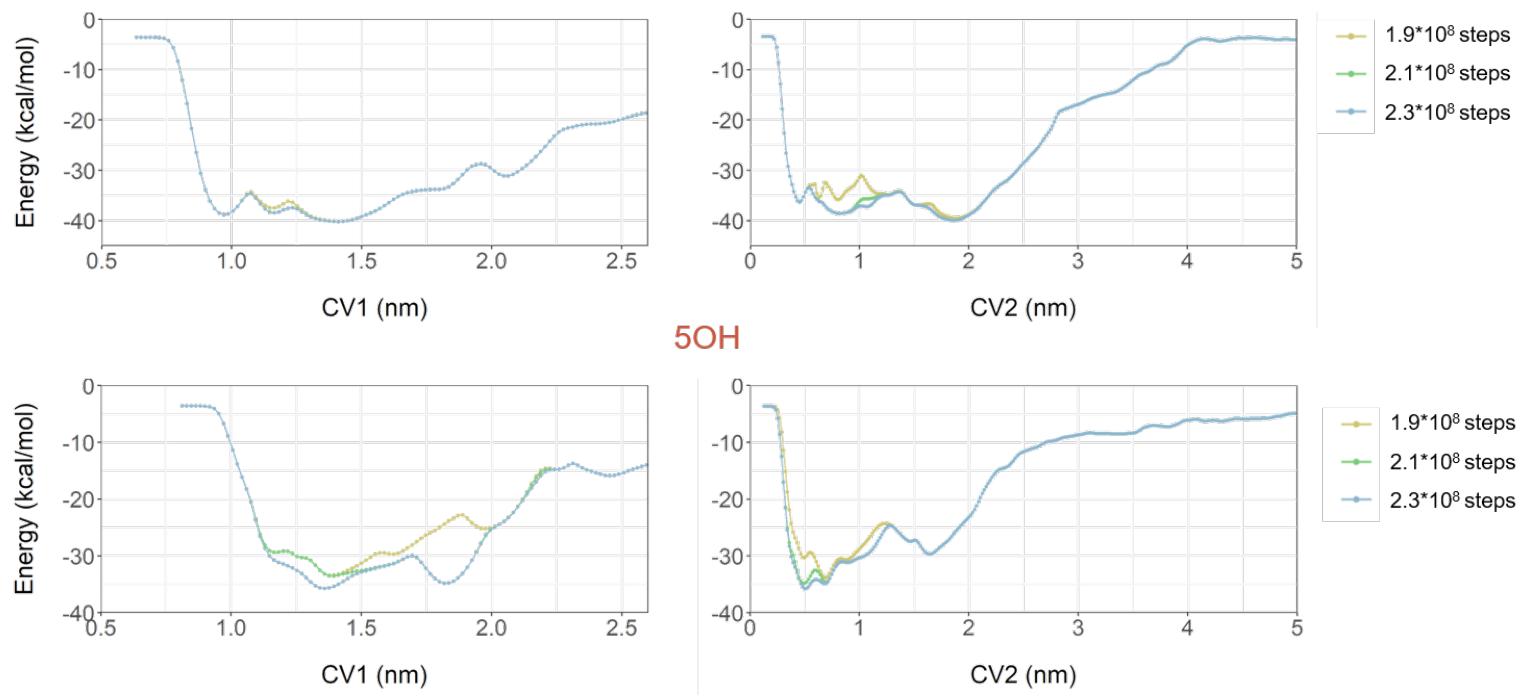

$5 \mathrm{OH}$

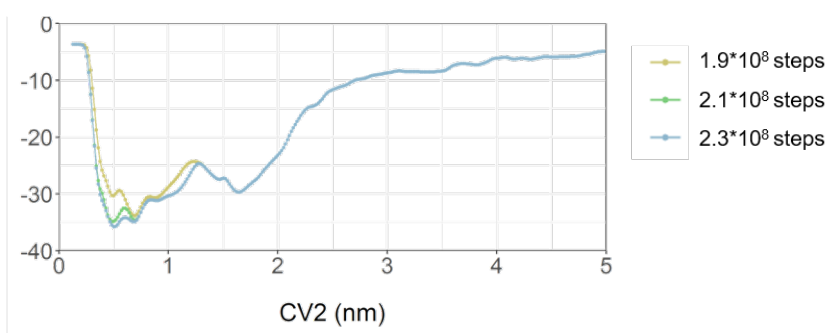

Figure S13. Convergence of the free energy profiles for the release of the leaving group from metadynamics simulations of the 5'-flap, 5PO and 5OH systems. Free energy profiles projected on CV1 (distance between the C.O.M. of the heavy atoms of the nucleotide leaving group and the C.O.M. of the C $\alpha$ of Glu160, Asp179, Asp181 - left panels) and on CV2 (distance between the C.O.M. of the nucleobases of $\mathrm{d} 1$ and the complementary nucleotide - right panels) are shown during the progress of the metadynamics simulations. 


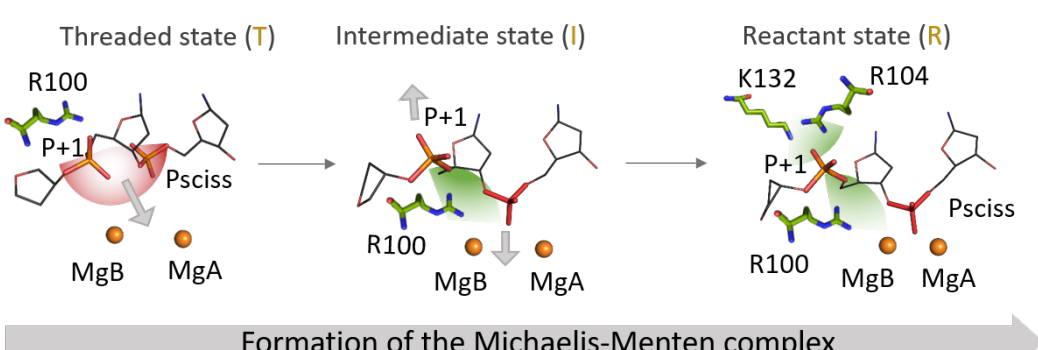

Formation of the Michaelis-Menten complex

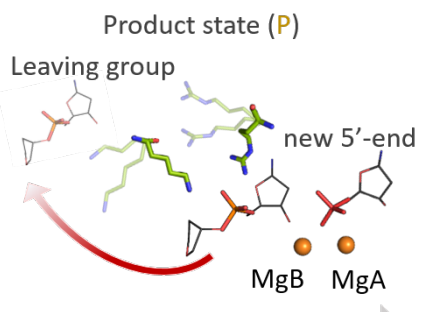

Leaving group release
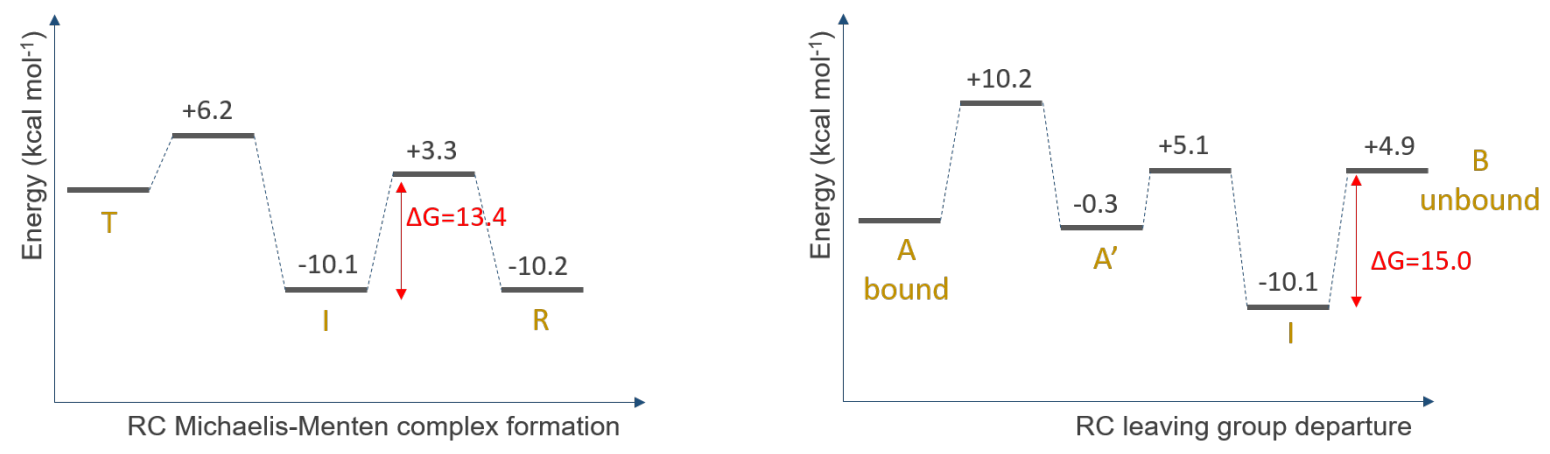

Figure S14. Energy profiles of the formation of the Michaelis-Menten complex and leaving group departure for the $w t$ hFEN1-5' flap substrate system. 


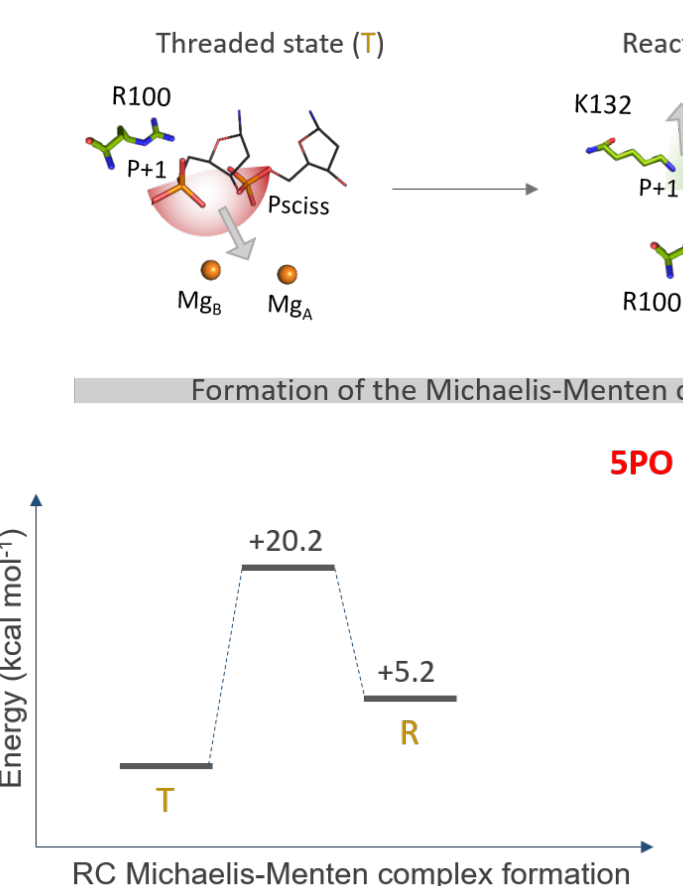

RC Michaelis-Menten complex formation

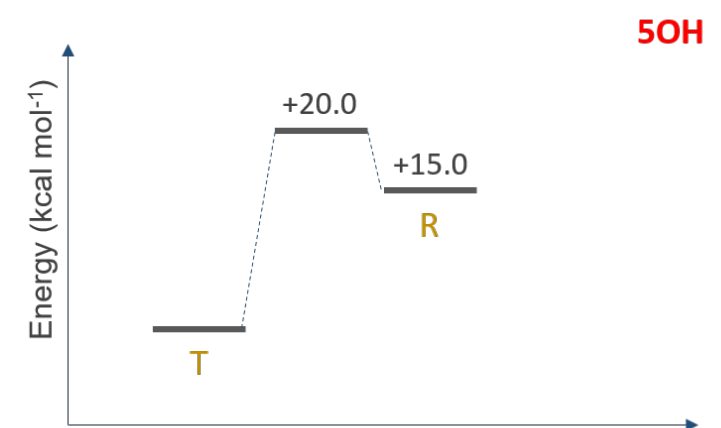

RC Michaelis-Menten complex formation

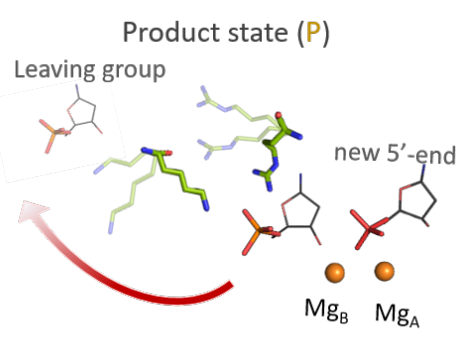

Leaving group release

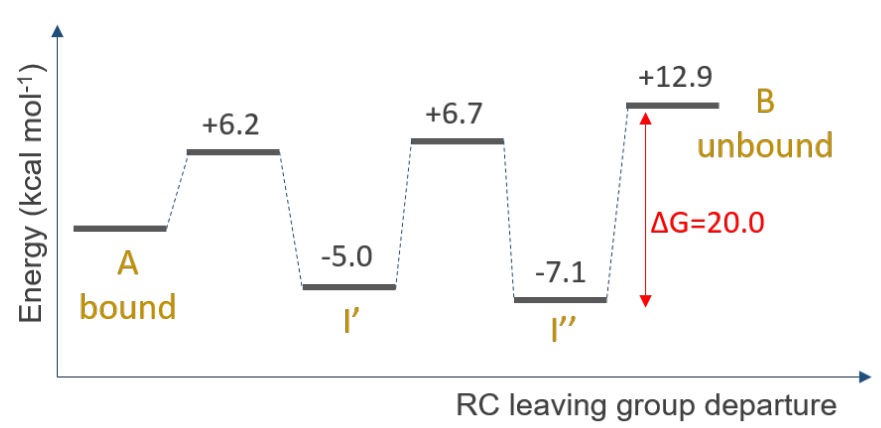

Figure S15. Energy profiles for the formation of the Michaelis-Menten complex and leaving group departure for both the $w t$ hFEN1-5' PO substrate system (5PO) and the $w t$ hFEN1-5'OH substrate system (5OH). 


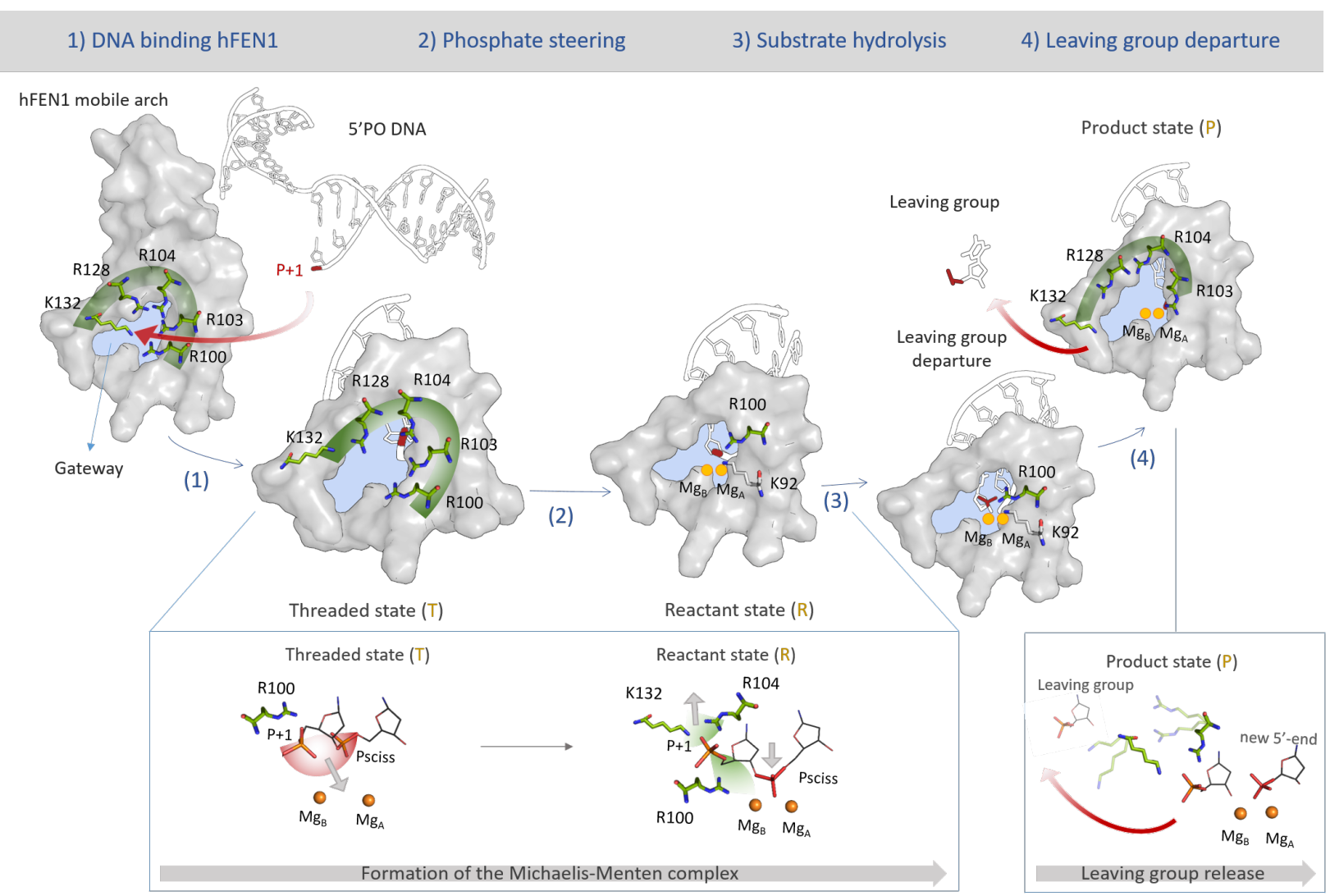

SchemeS1. Schematic representation of the catalytic steps during hFEN1 catalysis on exonucleolytic DNA substrate (i.e. 5'PO). On the bottom left box, the molecular mechanism of phosphate steering residues Arg104 and Lys132 acting concertedly with Arg100 to promote the Michaelis-Menten complex formation. In the bottom right box, the molecular mechanism for leaving group departure assisted by phosphate steering residues. 